\title{
Epistemología y \\ Teoría del Conocimiento \\ (a)
}

\author{
Epistemology and \\ Theory of Knowledge
}

Rolando García ${ }^{1}$

${ }^{1}$ Investigador Titular del
Centro de Investigaciones
Interdisciplinarias en
Ciencias y Humanidades, de
la Universidad Nacional
Autónoma de México,
CEIICH-UNAM.
rolandog@servidor.unam.mx
RESUMEN Este texto señala el origen del término epistemología, referido a la teoría del conocimiento científico. El tema está centrado en el desarrollo de la ciencia moderna y en particular en la crisis de los conceptos básicos a comienzos del siglo XX. Esto significó el fin de la filosofía especulativa en su capacidad para dar cuenta de las conceptualizaciones y de las teorías de la ciencia contemporánea. Al avanzar el siglo, también los más destacados empiristas debieron admitir su fracaso. La renuncia a las posiciones aprioristas y empiristas llevó a la necesidad de concebir el conocimiento como un proceso constructivo que al nivel individual se desarrolla desde el nacimiento hasta la edad adulta, y se prolonga al nivel social con el desarrollo de la ciencia.

PALABRAS CLAVE Epistemología; Filosofía; Ciencia; Conocimiento.

ABSTRACT This text refers to the origin of the term epistemology, which relates to the theory of scientific knowledge. The issue focuses on the development of modern science and, particularly, on the crisis of basic concepts in the early twentieth century. This meant that speculative philosophy was no longer able to account for contemporary scientific conceptualizations and theories. As the century drew on, the most outstanding empiricists also had to admit their failure. The rejection of aprioristic and empiricist positions resulted in the need to understand knowledge as a constructive process which evolves, at the individual level, from birth to adult age, and extends to the social level through scientific development.

KEY WORDS Epistemology; Philosophy; Science; Knowledge. 
La introducción de la última obra filosófica que publicó Bertrand Russell, sin duda una de las grandes cumbres de la filosofía del siglo XX, comienza así:

"Para el sentido común científico es obvio que sólo se conoce una parte infinitesimal del universo, que hubo épocas incontables en las que no existió ningún conocimiento y que, probablemente, habrá incontables edades futuras sin conocimiento; cósmicamente y causalmente, el conocimiento es un elemento sin importancia en el universo. Una ciencia que omitiera mención de su ocurrencia sólo padecería, desde el punto de vista impersonal, de una insignificante imperfección".

Es un estilo típico de Bertrand Russell para introducir uno de los libros más importantes sobre el conocimiento humano. Estamos de acuerdo en que es una parte infinitesimal del universo, pero es la parte más importante para nosotros, porque el conocimiento es sin duda la base de la vida de relación y, quizás lo más trascendente hoy en día, el conocimiento se ha convertido (más de lo que históricamente ha sido) en la base del poder.

Tratar este tema es bastante arduo. De acuerdo, somos una parte infinitesimal del universo, pero yo me tengo que ocupar forzosamente, en el tiempo de que dispongo, de una parte más infinitesimal todavía de este mundo terráqueo. Para tratarlo con cierta posibilidad de comprensión general, tendría que ocuparme del Asia, sobre todo del Asia Menor, de la China, de la India, de África, lo cual es prácticamente imposible. Voy a tener que hacer lo que es costumbre: omitir esa parte del mundo que ha sido un motor extraordinario en los problemas del conocimiento, con una visión muy distinta que la nuestra. Me voy a circunscribir a esta región espacio-temporal muy reducida que de manera muy arbitraria se llama mundo occidental, y a una parte temporal que es, como suele hacerse, de Grecia en adelante. Quizás haga, si me da el tiempo, alguna referencia, que siento obligada, para contraponer la visión que a lo largo de la historia desarroIlaron ambas civilizaciones.

Si empezamos con Grecia, la filosofía, la religión, la magia, la superstición y la ciencia empezaron mancomunadas, en un mundo de comprensión y de coexistencia. Con el advenimiento del Cristianismo el idilio terminó y vino la gran ruptura. San Agustín dijo que no se puede ser cristiano y filósofo al mismo tiempo "porque es vana la pretensión de la mente de llegar a verdad alguna: a la verdad se llega sólo por la revelación a través de la fe". El emperador Justantino lleva a la práctica las implicaciones de este dogma, cerrando la academia platónica, con el argumento de que allá "se imparten enseñanzas paganas y perversas". Hay un éxodo importante de los filósofos de Grecia y durante seis siglos no hay filosofía ni ciencia en Europa. Solamente la teología está autorizada a decir qué es conocimiento y qué es verdad.

Quienes emigraron de Grecia se fueron a Oriente. Por suerte para ellos no necesitaban tramitar pasaporte ni visa, así que pasaron directamente a Persia, a Jundi-Shapur, un centro que era originalmente un centro de medicina, y que fue adquiriendo un carácter de universidad. Pero cuando se fundó Bagdad (en el año 762) se concentró allí la elite científico-filosófica del mundo de entonces. Bagdad fue durante cinco siglos (algo para recordar frente a lo que pasa hoy), el centro intelectual del mundo. Allí dieron los árabes un ejemplo de tolerancia y libertad del pensamiento. Ahí estaban cristianos, judíos, árabes, y musulmanes conviviendo, rescatando y traduciendo las obras de la época del esplendor de Grecia. La ciencia heleno-árabe llegó a Europa a través de España, cuando los árabes fundan el Califato de Córdoba, cuya capital pasó a ser -según los historiadores- "la ciudad más poblada y más culta de Europa". Así vuelve la filosofía griega a Europa, en un momento en que, con la revolución agrícola, la expansión de las ciudades, el comercio, etcétera, etcétera, se produce ese extraordinario renacimiento intelectual que cambia la visión del mundo, en los siglos en que surgen las universidades.

Son cambios que atañen tanto a las relaciones con el mundo físico, como al tejido de relaciones en la sociedad. La Iglesia, cuya doctrina había quedado exclusivamente bajo el dominio de la teología, carecía de una filosofía que pudiera servir de intérprete de este tipo de cambios, con la efervescencia de ideas que ellos generan, y debe establecer nuevos marcos de referencia. Uno de ellos fue el mojón que plantó Tomás de Aquino (quién será después Santo Tomás), una de las grandes inteligencias de la 
iglesia cristiana. Fue él quien advirtió que no era posible mantener la total dominación de la teología en la interpretación de los fenómenos de este mundo terrenal, e introdujo la doctrina de "la doble verdad". El universo quedó dividido en dos dominios. Más arriba de la luna, era el dominio de la teología. Sólo ella podía decir qué eran los fenómenos, qué era la verdad. Por debajo de la luna se admitió que el hombre podía llegar a establecer algunas "verdades relativas" a través de la observación y la experimentación. En mi concepción de la historia de la ciencia, aquí se encuentra el germen de lo que será la actividad científica en el mundo occidental.

No voy a resumir la historia. Sólo mencionaré lo que todos saben; en los siglos siguientes, fundamentalmente con lo que se Ilama oficialmente el Renacimiento (siglos XV y XVI) se inicia un proceso social, económico, político y religioso que va a incluir las reformas de la Iglesia, que va a conducir a la revolución científica, y que culminará en la segunda parte del siglo XVII, con Newton. $Y$ me detengo en Newton porque el mundo newtoniano que va a dominar el resto del siglo XVII, todo el siglo XVIII, y que va a continuar en parte del siglo XIX, hace una ruptura absolutamente fundamental en el problema del conocimiento, que es el tema de estas reflexiones. Esa ruptura se concentra inicialmente en dos puntos.

Primer punto: se empieza a hablar por primera vez de leyes naturales. La palabra ley se usaba hasta entonces referida a normas morales o normas jurídicas. En la segunda mitad del siglo XVII (en la fecha precisa de 1665) y en lugar preciso (la publicación Philosophical transactions de la Royal Society) aparece por primera vez, (y seguirá usando de manera sistemática) el término "ley natural". La introducción de este término refleja el cambio fundamental que se produce, dentro del Protestantismo, con respecto a la concepción del mundo. El cambio, que yo llamo cambio de "marco epistémico", se refiere a lo siguiente: el mundo está creado por Dios, pero Dios estableció leyes y esas leyes rigen al mundo físico sin mediar más la voluntad de Dios. La implicación fundamental que tiene este cambio de doctrina para el desarrollo de la ciencia es la aceptación de que la mente humana puede desentrañar esas leyes. Jocosamente se dijo que Dios creó al mundo, le impuso sus leyes, y después mandó a Newton para que se las explicara al resto de la humanidad. El más ardiente seguidor de Newton, que fue Boyle, dirá que no solamente debe ser permitido que la mente humana estudie esas leyes, sino que es obligación del ser humano estudiar esas leyes para entender la armonía que Dios puso en el universo. El mundo que pinta la "filosofía natural" de los newtonianos incluye a la sociedad en su conjunto. Esas leyes "naturales" rigen también el orden económico, y una buena parte de la concepción de la economía que va a seguir después con el desarrollo del capitalismo será producto de ese pensamiento.

El segundo punto fundamental es la gran ruptura con la teología medieval y con la doctrina tomista de "la doble verdad". Newton muestra que las leyes que rigen los movimientos planetarios son las mismas leyes que rigen los movimientos aquí abajo, en el mundo sublunar. El movimiento de los planetas y el movimiento del péndulo obedecen las mismas leyes. Aquí termina la dictadura de la teología, que era el único tribunal autorizado a opinar cómo eran los fenómenos "más allá de la luna".

El hombre empieza a investigar el universo y a decidir acerca de la ciencia que está surgiendo, a decidir cuáles son los fenómenos de los cuales se va a ocupar, y a tratar de explicar esos fenómenos. Era natural que al mismo tiempo surgiera la revolución en la filosofía. Renace la filosofía. Es el comienzo de la filosofía moderna, y el padre de la filosofía como todos saben, es Descartes. Con el surgimiento de la filosofía moderna hay una especie de acuerdo tácito de dividirse la tarea. Para decirlo de manera un poco simplificada y quizá caricaturesca: la ciencia se va a ocupar de explicar al resto de la humanidad las leyes naturales, y la filosofía le va a explicar al científico qué es lo que sus teorías quieren decir. Salen de ahí los sistemas filosóficos. Salen de ahí naturalmente Locke y Hume, Berkeley y Leibniz, finalmente Kant. Ellos van a explicar qué es el espacio, qué es el tiempo, qué es la causalidad, qué son las matemáticas, qué son las teorías. Ellos son quienes les van a explicar a los científicos. Los científicos se ocuparán de las leyes y de desenmarañar esas leyes, pero no de decir qué son. Newton dice "yo no hago hipótesis" (sobre la naturaleza de la fuerza de gravedad). Pero su 
libro está impregnado de geniales hipótesis. En realidad todo su libro es una manifestación extraordinaria de lo que se llamará el método hipotético-deductivo, pero quizás no quiere comprometerse con afirmaciones que entraran en conflicto con la verdad religiosa, porque el espectro de la condenación de Galileo le andaba rondando y no quería tener problemas similares.

La culminación de todo este proceso es la filosofía kantiana. Kant viene de la ciencia empírica, es un físico, se ocupa también junto con Laplace, de la teoría de la nebulosa. Se ocupa de todo el mundo natural. Es poco conocido que Kant fue el primer profesor de geografía que hubo en el mundo. La primera cátedra de geografía que se abre en Alemania es para Kant. Un hombre genial que se ocupa de una multitud de temas. Su posición era empirista, viene de la física del siglo XVII, de Newton. Kant tropieza con Hume, empirista también, pero de posición muchísimo más flexible, y el más lucido analista de lo que pasa en la ciencia de entonces. Hume pone en tela de juicio todo lo que se ha dicho sobre causalidad. Todos los que hayan hecho algún curso de filosofía saben, habrán leído o habrán oído, el dicho de Kant de que Hume lo despertó de su "sueño dogmático", de creer solamente en los hechos. Bertrand Russell comenta con su habitual ironía: efectivamente, se despertó de su sueño dogmático, pero encontró pronto un soporífero que le permitió volver a dormir con toda placidez. El soporífero fue su propia teoría porque, a partir de esa puesta en duda de Hume, Kant elabora el más impresionante monumento, el más formidable sistema filosófico que se construyó, yo creo, en toda la historia de Occidente. Con respecto a él siempre repito el mismo (mal) chiste: es un sistema "casi" perfecto que tiene el defecto de ser falso.

El gran mérito que tuvo Kant entonces (y sigue siendo el gran mérito de Kant) es haber planteado con toda claridad el problema del conocimiento, el problema de la relación sujetoobjeto en la construcción del conocimiento; lo que ya no es aceptable son sus respuestas, que forman un sistema cerrado completo. Él explica el espacio, el tiempo, la causalidad, explica las matemáticas $y$, naturalmente, explica la ciencia de su época. Para él la geometría es lo que dicen los Elementos de Euclides; la lógica es el silogismo aristotélico; la matemática es el cálculo en la forma que Newton y Leibniz lo construyeron; el espacio y el tiempo es lo que Newton considera como espacio y tiempo. Él está convencido de que ha resuelto todos los problemas. Por eso se atreve a escribir, como coronación de su obra cumbre (La crítica de la razón pura), un complemento que lleva por título, modestamente, "prolegómenos a toda metafísica futura".

La obra de Kant es el monumento de la filosofía especulativa. Pero ese monumento tuvo mala suerte. Kant muere en 1804 y no pasan 20 años sin que la ciencia, fundamentalmente la matemática, tenga un vuelco extraordinario. Aparecen las geometrías no euclidianas, y a partir de ahí yo diría que cada uno de los conceptos que daba Kant como establecidos, va a ser sistemáticamente demolido en lo que resta del siglo y en el comienzo del siglo XX. La geometría no euclidiana muestra que la geometría de Euclides es sólo una de las geometrías entre otras equivalentes, y que la geometría del espacio físico no era un problema que podían decidir las matemáticas por sí mismas. Por su parte, la lógica va a ser completamente renovada en ese siglo. Se va a mostrar que la silogística de Aristóteles es sólo un pequeño capítulo de la lógica y se va a resolver lo que fue el gran escándalo de la matemática y de la lógica: la lógica de Aristóteles no es capaz de expresar al más simple razonamiento matemático, siendo que las matemáticas se consideran la cumbre del razonamiento lógico. Es fácil mostrar razonamientos muy simples que no son reducibles a silogismos. Cae entonces la lógica aristotélica. Weirstrass da al cálculo un aspecto completamente distinto: los infinitésimos que tanto le hicieron devanar los sesos a Kant y también a Hegel se muestra que no son problema. Y Cantor le resuelve las anatomías sobre el infinito. Brevemente se llega al final del siglo con una matemática distinta, sin que quede nada de los problemas de Kant.

En el principio del siglo siguiente, el siglo XX, con la relatividad y la mecánica cuántica, el proceso se va a terminar. El espacio y el tiempo cobran un sentido completamente distinto. Este es el derrumbe, no de Kant, no de Hegel, es el derrumbe de la filosofía especulativa. A partir de ahí la filosofía especulativa pierde el derecho de tratar de fundamentar los conceptos científicos. 
Los alemanes son los primeros que se percatan de eso, quizás porque una buena parte de lo que ocurrió, ocurrió en Alemania. Y lo que era erkenntniss theorie, la teoría del conocimiento (erkenntniss es conocimiento) le anteponen wissenshaft leherer, (wissenshaft es ciencia), le anteponen una teoría de la ciencia. Quien toma esto muy claramente y le da su sentido filosófico, quien retoma sobre todo la reconstrucción de la geometría, es Bertrand Russell, publicando en los últimos años del siglo XIX, una obra fundamental, Los fundamentos de la geometría, y ahí usa la palabra epistemology, como traducción o como el equivalente al wissenshaft leherer de los alemanes. No la teoría del conocimiento, no el erkenntniss, sino la teoría de la ciencia. El libro de Russell se traduce, poco después (en 1901), al francés y aparece ahí la palabra epistemoligie, que según el diccionario histórico de la lengua francesa es el punto de partida del uso de la palabra epistemología como distinta a la teoría general del conocimiento que había sido edificada por los filósofos. Quien nacionaliza el término epistemoligie, que va a pasar al español como epistemología, es Meyerson. El libro publicado poco después de Meyerson, Identidad y realidad, comienza su prólogo diciendo: "Me voy a ocupar de la filosofía de la ciencia o epistemología como hoy empieza a usarse". Es ahí el momento en el que aparece una epistemología como teoría de la ciencia, distinta a lo que la filosofía especulativa da como teoría del conocimiento. Entonces, a partir de ese momento se hace necesario distinguir entre una teoría del conocimiento que podríamos Ilamar teoría del conocimiento común, y una teoría del conocimiento científico que sería la epistemología.

Bueno, pero ¿qué pasa entonces con el conocimiento científico? Es cierto, la ciencia ha demostrado que las disciplinas se han renovado, que los conceptos tradicionales que los filósofos habían analizado han caducado por completo. Cabe preguntarse entonces ¿qué imagen del mundo da la ciencia?

Un libro de sir Arthur Eddington que fue muy difundido, best seller cuando yo era joven, formuló el problema de manera impactante. Eddington fue el primero que dio pruebas empíricas de las teorías de Einstein cuando, en una famosa expedición organizada por la Royal Society para observar un eclipse total de sol en
Brasil, encontró que efectivamente los rayos de luz de una estrella se curvan al pasar cerca del sol. Su libro, La Naturaleza del Mundo Físico, plantea lo que se llamó "el problema de las dos mesas". Yo estoy trabajando sobre esta mesa, pero en realidad hay dos mesas. Está frente a mí esta mesa sólida, que tiene un color determinado, que tiene un peso determinado, que es donde yo me apoyo cuando estoy trabajando. Pero la física me dice que esta mesa tiene una materia que está compuesta por moléculas y que las moléculas están compuestas por átomos y que los átomos tienen partículas y que todos los elementos están en revolución y muy separados entre sí. Eddington agrega, y ésta es la frase crucial: si pudiéramos juntar las partículas del átomo, juntar los átomos, juntar las moléculas, el total de la materia de esta mesa cabría en la punta de un alfiler. Entonces, se pregunta, ¿qué es la mesa?, ¿es esta mesa donde yo me apoyo o es lo que nos dice la física? Bertrand Russell con su estilo extraordinario traduce esto en sus obras diciendo: "El realismo ingenuo nos hace aceptar los objetos del mundo tal como parecen, aceptar que ahí está la mesa, y están las sillas como las vemos. El realismo ingenuo nos conduce a la física, pero la física nos da una imagen que contradice al realismo ingenuo. Si la física es cierta, el realismo ingenuo es falso". Esto se tomó como una humorada, como una de las famosas ironías de Russell. Quien no lo tomó así fue Einstein, quien contribuyó, quizás más que nadie a la "imagen" que tenemos hoy del mundo físico. Einstein toma esa boutade de Russell y dice "éste es el problema fundamental". Naturalmente que Einstein creía en la física, para él la física es comprobable, es así. Pero entonces ¿cómo paso de este mundo de sensaciones, de este mundo perceptual, al mundo de las teorías físicas? Bueno, Einstein da su versión, la cual no voy a comentar, voy a dejarla completamente de lado, porque ese no es el Einstein que construye las teorías sino el Einstein que las interpreta, y ahí entra una concepción del mundo muy religiosa, con la cual habría que construir otros puentes que Einstein deja sin aclarar. Es ése el momento en que empieza a plantearse esa problemática de la cual solo puedo ofrecer aquí un muy ligero esbozo cuando surgen las escuelas empiristas de principios del siglo XX, que son escuelas absolutamente extraordinarias, 
no solamente por lo que van a hacer en ciencia, sobre todo en el desarrollo de la lógica y de la matemática, sino porque se plantean con todo rigor el problema que acabo de exponer.

El problema fundamental es cómo se pasa de las sensaciones a la construcción de las teorías. El problema que propone el empirismo lógico lo formula de manera muy nítida y con total coherencia con su posición epistemológica: si el empirismo es correcto, todo lo que dicen las teorías puede finalmente ser expresado en término de sensaciones y de relaciones entre las sensaciones. La escuela de Viena, con Carnap a la cabeza, se plantea el problema de llevar a la práctica una investigación muy concreta: muy bien, vamos a empezar con las sensaciones y vamos a construir los conceptos físicos. Este es para mí uno de los grandes experimentos epistemológicos de la historia de la humanidad. El libro en el cual se exponen los resultados es La estructura lógica del mundo, un libro absolutamente extraordinario de uno de los grandes lógicos del siglo XX. Carnap tiene que confesar que ha fracasado, no puede pasar de las sensaciones a construir los conceptos de la física.

La segunda experiencia, dentro de un programa similar la hace Bertrand Russell con un método completamente distinto. Bertrand Russell parte del lenguaje de la ciencia (el lenguaje de la física) e intenta reducirlo a un vocabulario mínimo. Entiende por vocabulario mínimo un vocabulario tal que todas las proposiciones de la física pueden ser expresables estrictamente en los términos de su vocabulario, pero que además ningún término del vocabulario sea definible por los otros. Empieza a trabajar con vocabularios mínimos que tengan referentes directos en las percepciones y se propone, a partir de allí, construir los conceptos de la física. Segundo fracaso, que Russell hace explícito: no podemos, a partir de proposiciones que representan nuestras sensaciones construir un vocabulario suficiente para la ciencia, porque faltan las relaciones, y las relaciones no son observables, ni son reducibles directamente a observables. Ésa es la segunda gran experiencia epistemológica.

El último de los libros filosóficos de Russell, El conocimiento humano, termina con un capítulo que se titula "los límites del empirismo", y en el último párrafo de ese último capítulo afirma: "debemos confesar que el empirismo como teoría del conocimiento es inadecuado", y agrega, quizás como consuelo, "pero es mejor que todas las anteriores y no tenemos otra cosa". Ésa es la última confesión de Russell: los límites del empirismo.

Ha habido muchos otros que hicieron estos intentos. El tercero que voy a mencionar, el que siempre menciono, es Quine. Quine es uno de los grandes lógicos vivientes, y empirista a carta cabal toda su vida, que también trata de mostrar cómo se pasa de las sensaciones a los conceptos científicos. Y lo que yo llamo "el certificado de defunción del empirismo" lo firma Quine en el congreso de filosofía de Viena donde dice una frase que es extraordinaria para quien fue el gran positivista del siglo: "hemos dejado de soñar en construir una ciencia a partir de los datos de los sentidos". Escrito por Quine.

Estas singulares experiencias dan a este período un carácter absolutamente extraordinario en la historia de la filosofía, porque no creo que haya otro período en el cual realmente se intente llevar a sus últimas consecuencias una posición filosófica.

Entonces, ¿en qué queda el problema después de la defunción del empirismo? Recapitulemos. A principios del siglo había tenido lugar lo que yo llamo el primer derrumbe epistemológico del siglo XX, cuando la filosofía especulativa debe renunciar a fundamentar los conceptos de la ciencia. Luego viene, a mediados del mismo siglo XX, lo que yo llamo el segundo derrumbe epistemológico del siglo, que es la evidencia de la insuficiencia del empirismo para fundamentar los conceptos científicos. ¿Qué es lo que queda? La consecuencia práctica ha sido $-\mathrm{y}$ esta es una posición personal, una opinión muy personal que voy a expresar de manera un tanto osada-: lo que hoy se llama filosofía de las ciencias en las universidades, en las facultades, en los textos, carece de fundamentación epistemológica. La filosofía especulativa no pudo fundamentar la ciencia, el empirismo tampoco. La ciencia se quedó sin epistemología. Fíjense ustedes Kuhn, Feyerabend, Lakatos, y el mismo Popper, no hacen epistemología, no muestran como se genera el conocimiento, se acabó ese tipo de investigación. Lo que hacen es nada más, ni nada menos, que sociología de la ciencia. 
¿Cuáles serían las consecuencias reales, para la investigación, al haber renunciado al apriorismo de la filosofía especulativa y al empirismo? La respuesta la encontré cuando tuve la enorme fortuna de poder colaborar con Piaget. En ese libro que se llama Psicogénesis e historia de la ciencia (b), mostramos lo que Ilamamos "mecanismos comunes".

Hablamos de "mecanismos comunes", porque hicimos comparaciones entre cómo se generan los conceptos en la psicogénesis en los niños y como se generan los conceptos en la ciencia. Y encontramos que los mecanismos últimos, no los resultados del proceso cognoscitivo, no las estructuras que se generan, sino los mecanismos últimos, eran comunes. Yo creo ahora que el problema hay que plantearlo de una manera distinta de como lo planteamos en ese libro. Si tenemos que renunciar a conceptos a priori, si tenemos que renunciar a los datos de los sentidos como origen del conocimiento, quiere decir que en todo el transcurso del conocimiento, desde el nacimiento hasta la ciencia, no pueden haber discontinuidades funcionales, porque si hubiera una discontinuidad funcional, si hubiera un "antes" y un "después" en alguna parte del conocimiento, entonces volvería a plantearse el problema de cómo se basa el antes y el después; ¿otra vez por conceptos a priori?, ¿otra vez por datos de los sentidos? Si hay discontinuidad, significaría replantear allí el problema del apriorismo y del empirismo. Debemos aceptar, por consiguiente, una continuidad en el conocimiento, sin comienzo (sea el conocimiento o las actividades que podemos Ilamar cognoscitivas). Esto significa que esas actividades están incluso antes del nacimiento, se sumergen en la biología, y que hay un continuo desde la biología al desarrollo de las actividades que luego van a ser cognoscitivas. Significan, además, que esas actividades del niño, del adolescente, del adulto no sofisticado, tienen continuidad con la ciencia, que hay una continuidad funcional de mecanismos en todo ese proceso.

Y eso para mí -aquí expreso una opinión personal- es independiente de toda posición filosófica. Esto es lo que está implícito en el constructivismo piagetiano. Es lo que sostuvo Piaget, sin haberlo dicho así. Para mí, la renuncia al apriorismo y la renuncia al empirismo supone o implica aceptar la continuidad del proceso cognoscitivo.
Entonces se replantea desde allí el problema de ¿en qué consiste el conocimiento? Contestar que el conocimiento es una construcción, no resuelve el problema ¿qué se construye? ¿cómo se construye? No construimos los objetos, no construimos las mesas, casas, ¿qué es lo que construimos? Ahí sí tengo yo que recurrir a todas las investigaciones psicogenéticas que se han hecho durante 60 o 70 años en la escuela ginebrina. Lo que se construye es la forma de organizar las interacciones con el mundo externo. El niño que nace con reflejos innatos, que nace chupando (porque los que no chupan se murieron), que nace con ese reflejo de succión, que nace con el reflejo palmario, que patalea, ese niño poco a poco va organizando sus movimientos, entra en una interacción con el mundo, y yo creo que la gigantesca tarea que hizo la escuela de Ginebra fue ir mostrando paso a paso en qué consistió la organización de esas interacciones a partir de las cuales se genera el conocimiento. Conocer es organizar los datos de la realidad, darles un sentido, lo cual significa construir una lógica, no la lógica de los textos, sino una lógica de la acción, porque organizar es estructurar, es decir, hacer inferencias, establecer relaciones. Estructura es lógica. Volvemos a un estructuralismo, pero que no tiene nada que ver con los estructuralismos clásicos. Es un estructuralismo que llamamos "genético" en el sentido de concebirlo como la génesis del conocimiento a través de organizaciones estructurantes. A Piaget se le ha condenado como estructuralista, pensando que de alguna manera reflejaba las polémicas sobre el estructuralismo que hubo a mediados del siglo XX. Lo que se olvida es que para Piaget (lo voy a decir de manera un poco paradójica), no se trata de un sustantivo, "estructura", se trata de un verbo, "estructurar". Se trata de organizar nuestra experiencia, y esa organización es crear estructuras. No es posible que ahora intente de ninguna manera explicar cómo ocurre.

En síntesis, el problema del conocimiento empezó a tratarse de una disciplina que se ocupa de todo el conocimiento: tanto del conocimiento infantil, como del hombre adulto "normal", para pasar a las actividades científicas. Tal fue el dominio de la filosofía especulativa. Sin embargo la filosofía especulativa tuvo que retroceder cuando todas las cosas que afirmó fueron 
contradichas por la ciencia, no por otro sistema filosófico, sino por la ciencia. No sólo retroceder, sino dejar parte de su campo a los científicos.

Con el empirismo se realizaron notables avances en problemas de fundamentación de las ciencias. Pero cuando trataron de fundamentar el conocimiento sobre bases estrictamente empiristas, invadieron de hecho territorios que la filosofía consideraba como propio. Más aún, al declarar que todo conocimiento surge de la experiencia y que las afirmaciones que no son directa o indirectamente reducibles a proposiciones referidas a datos sensoriales no pueden tener sentido cognoscitivo, realizan una amputación de una parte considerable de la filosofía.

Esta fue, sin embargo, una situación transitoria. El fracaso del programa empirista, que hemos señalado, significó un "regreso" de la filosofía, en una nueva fase del movimiento pendular que caracterizó las relaciones entre la ciencia y la filosofía a lo largo de la historia.

Hoy tenemos ideas más claras sobre este problema, porque contamos con una teoría que nos permite concebir el conocimiento como un proceso continuo que, al nivel individual, se desarrolla desde el nacimiento hasta la edad adulta, e incorpora al nivel social el desarrollo de la ciencia.

Es una teoría del conocimiento en la cual los procesos cognoscitivos no tienen más punto de partida que las raíces biológicas del individuo y sus interacciones con el mundo en el cual actúa.

Incorporar las raíces biológicas a la teoría del conocimiento significa reconocer una frontera móvil que los enormes progresos de la neurofisiología han ido desplazando, mostrando que muchos aspectos del comportamiento individual que se consideraba pertenecían a un terreno totalmente ajeno a la biología tienen en realidad explicación biológica. Esto no da pie para sustentar alguna forma de reduccionismo. Para la teoría epistemológica constructivista, el desarrollo del conocimiento aún en los niveles más fundamentales, reclama otros elementos constructivos.

En la brevísima síntesis precedente hemos utilizado repetidamente el término "conocimiento" sin intentar definirlo, por la simple razón de que no hay definición de conocimiento. Contrariamente a lo que sostuvo el positivismo, ninguna disciplina comienza con definiciones.
Esto ya lo sabía Newton, quien soslaya el problema de las definiciones iniciales en sus famosos Principia, declarando: "yo no defino tiempo, espacio, lugar y movimiento porque son bien conocidos por todos". Está claro que toda la teoría revolucionaria que allí expone Newton es teoría del movimiento, pero advierte que no necesita definir el término "movimiento". Le basta con definir "transformaciones del movimiento". Tampoco los matemáticos definen "número". Claro que se ocupan de los números -pueden definir lo que es un número natural, un número racional, un número real- pero el término "número" aisladamente, no se define ¿cómo empezamos, entonces, a tratar el conocimiento, la ciencia?

Aquí me referiré nuevamente a la escuela de Ginebra. Piaget caracteriza la ciencia como una institución social, lo cual significa que cada sociedad, en cada momento histórico, define ciertas actividades como actividades cognoscitivas, y designa el producto de esas actividades como conocimiento. El conocimiento, y en particular el conocimiento científico, es un producto social, y no tiene más definición que la que le otorga el contexto social en el cual se genera.

Esta posición la hemos explorado con Piaget en Psicogénesis e Historia de la ciencia, y he procurado profundizarla en El conocimiento en construcción (1), precisando más su sentido: la ciencia que se produjo en distintas culturas respondió no solamente a mecanismos internos del desarrollo del conocimiento, sino también a las características de la cultura en la cual se desarroIló. Mi principal punto de referencia ha sido Oriente, y en particular China.

Lo que fue China como civilización recién se conoció en Occidente en el siglo XX. La concepción que hubo en el siglo XIX era deformada y errónea. Incluso algún gran pensador que habla de las ciencias como un producto puramente occidental, y me refiero a Max Weber, hace afirmación con la visión que el siglo XI hace de China. Hoy sabemos que no es el caso que la China se haya simplemente atrasado con respecto a Occidente, sino que tenía una concepción del mundo muy distinta. Me atrevo a decir que la concepción del mundo que tenían los chinos, y más precisamente el taoísmo, fue una concepción que se desechó en Occidente sin comprenderla. Hubo excepciones. La más notable fue la filosofía 
organicista de Whitehead, con su antecedente en Leibniz de quien se sabe que recibió la influencia de los jesuitas que trajeron el taoísmo de China.

El mundo chino era un mundo en devenir, un mundo de permanente cambio. Y era también un mundo que actuaba como un organismo (con la imagen de nuestro propio organismo), que actúa como una totalidad que no es parcializable. Esta concepción organicista genera un pensamiento dialéctico contrapuesto a la concepción atomística, característica del mundo occidental, y el atomismo ha condicionado de diversas maneras el desarrollo de las disciplinas, aun aquellas que son puramente formales. Daré solo como ejemplo el atomismo lógico de Bertand Russell, el cual conformó en gran medida la manera de abordar la lógica en nuestro sistema de enseñanza, comenzando por la lógica proposicional, es decir, con enunciados o proposiciones elementales, que se llamaron "proposiciones atómicas", las cuales se asocian entre sí por medio de "conectivos lógicos", formando proposiciones "moleculares" cuya validez se analizaba con "las tablas de la verdad". El lenguaje mismo en que se expresó la "lógica moderna" reflejó claramente el contexto conceptual que le dio origen, y las conocidas paradojas a las que conduce muestran la debilidad para fundamentar la lógica. Hoy sabemos que hay maneras diferentes de plantear la lógica. El análisis psicogenético, desde una percepción epistemológica constructivista ha puesto en evidencia una lógica de la acción y una lógica de la significación, de base inferencial, que precede al razonamiento proposicional y que es próximo a la concepción dialéctica del conocimiento, tema que hemos expuesto con Piaget en el libro Hacia una lógica de significaciones (2).
Después de este panorama, que deja muchas lagunas y temas truncos quedará flotando el interrogante: ¿pero entonces en qué consiste la ciencia? Las respuestas tienen una multiplicidad de variantes que rebasan las formulaciones académicas. Podemos tomar como ejemplo lo que escribió a principios del siglo XIX el más grande de los paisajistas ingleses. Constable afirmó que la pintura es una ciencia, y que las pinturas -los cuadros pintados- son experimentos. Sin duda un músico podría haber dicho algo similar. Este tipo de afirmaciones que pudieron quedar como expresiones de artistas un tanto superficiales, fueron retomadas por un filósofo de la ciencia no de segundo orden: Nelson Goodman. En un libro provocador, The ways of world making (la forma de hacer, construir el mundo) Goodman contrapone las consideraciones puramente racionales, con otras maneras de concebir el mundo. Se podrá replicar que, si se utiliza el término "ciencia", debe comenzarse por hechos, por constataciones, por verificaciones. En este contexto pienso que vale la pena leer a Hilary Putnam, quizás el filósofo norteamericano hoy más prominente. A este respecto recordemos el problema que se planteó el positivismo: ¿cuál es el lugar de los valores en el mundo de hechos? El mundo es un mundo de hechos. ¿Cómo surgen los valores? Putnam da vuelta al problema y pregunta: ¿cuál es el lugar de los hechos en un mundo de valores? Porque el mundo en el cual actuamos es un mundo de valores.

Me detengo aquí porque se acabó el tiempo que me asignaron, y tengo una excelente excusa para no entrar por esos derroteros.

\section{NOTAS FINALES}

a. Texto de una conferencia del seminario Formación y Reestructuración de Conceptos en Ciencias y Humanidades realizado en el Centro de Investigaciones Interdisciplinarias en Ciencias y Humanidades, de la Universidad Nacional Autónoma de México, CEIICH-UNAM. Editado en video y publicado por la misma institución. b. La edición original está publicada en español por Siglo XXI, y en francés por Flamarion. Hay traducciones al italiano, el inglés, el portugués, el japonés y el chino. 


\section{REFERENCIAS BIBLIOGRÁFICAS}

1. García R. El conocimiento en construcción. De las formulaciones de Jean Piaget a la teoría de sistemas complejos. Barcelona: Gedisa; 2000 .
2. Piaget J, García R. Hacia una lógica de significaciones. Serie Lógica y Epistemología, Bibliotecas Universitarias. Buenos Aires: Centro Editor de América Latina; 1998. [Edición original: Vers une logique des significatio. Ginebra: Murionde; 1987]

FORMA DE CITAR

García R. Epistemología y Teoría del Conocimiento. Salud Colectiva. 2006;2(2):113-122.

Recibido el 27 de febrero de 2006

Versión final presentada el 20 de marzo de 2006

Aprobado el 29 de marzo de 2006 